\title{
Correction to: Autonomy-induced preference, budget reallocation, and child health
}

\section{Biswajit Mandal $^{1} \cdot$ Prasun Bhattacharjee $^{2}$ (1) $\cdot$ Souvik Banerjee $^{3}$}

Published online: 9 August 2018

(c) Eurasia Business and Economics Society 2018

\section{Correction to: Eurasian Econ Rev https://doi.org/10.1007/s40822-018-0091-7}

When we published this article, the Assumption 1 on page 4 contained an error: while the preceding paragraph stated:

Mother's autonomy or the degree of empowerment is captured by making the parameters of the utility function dependent on mother's autonomy coefficient $\alpha$ such that $0 \leq \alpha<1$. Specifically, $\gamma=\gamma(\alpha)$ and $\delta=\delta(\alpha)$ where $0<\gamma(\alpha)<1$ and $0<\delta(\alpha)<1$ are the utility elasticities with respect to $H_{C}$ and $G$, respectively.

The following Assumption stated the contrary:

Assumption $1 \gamma^{\prime}(\alpha)>0$ and $\delta^{\prime}(\alpha)>0$

This mistake happened during typesetting and sadly remained unnoticed. Assumption 1 should read correctly:

Assumption $1 \gamma^{\prime}(\alpha)>0$ and $\delta^{\prime}(\alpha)<0$

The article has been updated. The publisher apologises for this mistake.

The original article can be found online at https://doi.org/10.1007/s40822-018-0091-7.

Prasun Bhattacharjee

bhattacp@etsu.edu

Biswajit Mandal

biswajiteco@gmail.com

Souvik Banerjee

souvikb@bu.edu

1 Department of Economics and Politics, Visva-Bharati University, Santiniketan 731235, India

2 Department of Economics and Finance, East Tennessee State University, Johnson City, TN 37614, USA

3 Boston University School of Medicine, Boston, MA 02215, USA 